\title{
Positive Growth of Phytoplankton under Conditions Enriched with Steel-making Slag Solution
}

\author{
Yasue NAKAMURA, Akira TANIGUCHI, Shigeru OKADA ${ }^{1)}$ and Masanori TOKUDA ${ }^{2)}$ \\ Division of Environmental Bioremediation, School of Agriculture, Tohoku University, Tsutsumidoori-Amamiyamachi, Aoba-ku, \\ Sendai, Miyagi-ken, 981-8555 Japan. \\ 1) Institute for Advanced Materials Processing, Tohoku University, Katahira, \\ Aoba-ku, Sendai, Miyagi-ken, 980-8577 Japan. \\ Aoba, Aoba-ku, Sendai, Miyagi-ken, 980-8578 Japan. \\ 2) Center for Interdisciplinary Research, Tohoku University, Aramakiaza
}

(Received on August 4, 1997; accepted in final form on December 15, 1997)

\begin{abstract}
The growth ability of 12 species of phytoplankton was examined in culture media enriched with steel-making slag solution at various concentrations. Since a highly significant linear regression of the in vivo fluorescence (IVF) of cell suspension against absolute chlorophyll a concentration was established, the growth ability was determined quickly by IVF. Under the enriched conditions all the species examined showed positive growth, although in different manners. Based on the specific differences in the optimal concentration of the slag solution, 12 organisms could be divided into four groups: (I) the organisms which grow best under the highest enrichment (100\%), (II) those which grow best with intermediate enrichment, but not in $100 \%$ enrichment, (III) those which grow best under enrichment lower than $60 \%$, and (IV) those which respond inconsistently to enrichment but often grow better under enrichment. These results indicate that the slag solution has positive effects for the growth of phytoplankton, so that the slag will be utilized as nutrients in cultures for algae. On the other hand, the growth of all the four groups enhanced by the enrichment with the slag solution never exceeded the growth enhanced in an ideal culture medium, indicating that the enrichment with the slag solution can hardly be a cause of the red tide, if the enrichment is done in natural seawater.
\end{abstract}

KEY WORDS: growth enhancement; in vivo fluorescence; phytoplankton; steel-making slag; waste products.

\section{Introduction}

The capability of oceanic waters to support the production of phytoplankton populations is expected to be enhanced by increasing the level of bioactive trace metal concentrations. It is reported that among the bioactive trace metals iron is the most important element for phytoplankton growth, while the other bioactive trace metals can be possible inhibitors of phytoplankton growth in the absence of organic ligand. ${ }^{1-7)}$

On the other hand, the residual production of steelmaking slag is huge. For example, in Japan today about 10 megatons a year are produced and used only for reclamation. Since the steel-making slag contains some effective elements of phytoplankton growth such as iron, silica and phosphorus, in the future, it could be used on an intensive culture of algae as nutrients and on a large scale to enhance production of the upper oceanic ecosystems. ${ }^{8)}$ It is very important to confirm whether cultures grow positively or negatively when the steel-making slag being employed as nutrients for phytoplankton culture.

In this work we preliminarily assayed the growth enhancement in phytoplankton in laboratory cultures with the addition of the steel-making slag solution at various concentrations.

\section{Method and Material}

\subsection{Selection of Basal Seawater for Bioassay}

Since it is necessary to use seawater with a known chemical composition to prepare the basal medium, we selected the artificial seawater called Marine Art (Senjyu Seiyaku Co.). This selection made the growth enhancement of the slag solution at different concentrations easy to determine. Prior to the experiments we tested the growth of the 12 phytoplankters which would be used in the follow-up experiments by putting them into a culture medium prepared in the Matsudaira's formula based on both artificial seawater and natural seawater. Although all the phytoplankters grew significantly in both media, most phytoplankters grew better in the medium prepared with natural seawater. This indicates that artificial seawater is free from both some growth factors as well as unknown growth inhibitors which might be present in natural seawater.

\subsection{Slag Solution}

For the above reason, we used artificial seawater to make the basal solution of the steel-making slag. The slag was ground into small particles which passed through 
a $1.7 \mathrm{~mm}$ mesh screen, yet retained on a $0.1 \mathrm{~mm}$ mesh screen. The particles were washed with distilled water in a beaker without shaking for $30 \mathrm{~min}$ and then transferred to the artificial seawater. These in turn were kept shaking at $20^{\circ} \mathrm{C}$ for 10 days to obtain the saturated concentration of the slag solution. The increased $\mathrm{pH}$, mainly due to free lime dissolved from the slag, was adjusted to $\mathrm{pH} 8$ by adding $\mathrm{HCl}$ every day. After about 10 days, stability at $\mathrm{pH} 8$ was usually maintained for at least half a day, and the solution was then filtered through a Whatman $\mathrm{GF} / \mathrm{F}$ filter $(0.7 \mu \mathrm{m}$ pore size) to remove residual particles. The chemical composition of the slag solution used to prepare later culture media was determined with an inductively coupled plasma spectrometer Model SPS-1200A (Seiko Instrument Inc.). To determine this, coprecipitation of trace elements in solution with zirconium hydroxide was used to retain the elements such as $\mathrm{Na}, \mathrm{K}, \mathrm{Mg}, \mathrm{Ca}$ etc.

\subsection{Cultivation}

Twelve species were employed to estimate the growth enhancement capability of the slag solution. A clone of haptophyte Isochrysis galbana was obtained from the culture collection of Dr. K. Shiraishi at the Department of Biology of the Iwate Medical College; those of cryptophyte Rhodomonas lens, chlorophyte Dunaliella tertiolecta and cyanophyte Synechococcus sp. from the Woods Hole Oceanographic Institution; chlorophyte Tetraselmis tetrathela from the collection of the National Institute of Aquaculture; haptophyte Emiliania huxleyi from the collection of Dr. Inoue at the Tsukuba Uni- versity; bacillaliophyte (diatom) Chaetoceros gracile, Skeletonema costatum and Thalassiosira angulata from our own collection; diatom Thalassiosira allenii, dinophyte Amphidinium carterae and chlorophyte Chlorella sp. from waters of Onagawa and Matsushima Bays, Miyagi Prefecture, during this work. Stock cultures were maintained in $30 \mathrm{ml}$ vessels containing about $15 \mathrm{~m} l$ of the Matsudaira medium under the conditions of $20^{\circ} \mathrm{C}$ and a $14: 10 \mathrm{LD}$ photocycled fluorescent light of about $47 \mu \mathrm{E} \mathrm{m}^{-2} \mathrm{~s}^{-1}$.

\subsection{Growth Condition and Media}

The medium for the following growth experiments was prepared by diluting the saturated slag solution (100\%) with artificial seawater to $20,40,60$ and $80 \%$. As controls at both extremes, growth was also determined in untreated natural filtered seawater, artificial seawater, and in culture media made of both natural and artificial seawaters by adding an ideal nutrient mixture prepared according to the Matsudaira's formula. ${ }^{9}$ In addition, because steel-making slag is almost completely free from nitrogen, artificial seawater as a control and the slag solution at different concentrations were enriched with nitrogen source and with a mixture of nitrogen, phosphorus and vitamin sources. The data on the culture experiments testing growth ability of 12 phytoplankton are summarized in Table 1 .

All of the media were sterilized by being filtered through a $0.20 \mu \mathrm{m}$ DISMIC-25cs filter unit (Advantec Toyo Inc.) immediately before use. The original inoculum was $1.0 \mathrm{ml}$ of a stock culture at the logarithmic growth

Table 1. General information on the culture series for testing the growth ability of 12 phytoplankters under enriched conditions with a steel-making slag solution (for Lot No. cf. Table 2). The following abbreviations were used: MA (artificial seawater), SW (natural seawater), MAE (artificial seawater enriched with nutrients), SWE (natural seawater enriched with nutrients), MAA (artificial seawater added only with nitrogen) and MAABV (artificial seawater added with a mixture of nitrogen, phosphorus and vitamins).

\begin{tabular}{|c|c|c|c|c|}
\hline Species & Lot & $\begin{array}{l}\text { Concentration of slag } \\
\text { solution* } \\
(\%)\end{array}$ & Control & $\begin{array}{c}\text { Duration of } \\
\text { incubations } \\
\text { (days) }\end{array}$ \\
\hline Skeletonema costatum & SL-4 & $\begin{array}{l}100,80,60,40,20 \\
100 \mathrm{~A}, 60 \mathrm{~A}, 100 \mathrm{ABV}, 60 \mathrm{ABV}\end{array}$ & $\begin{array}{l}\text { MA, SW, MAE, SWE } \\
\text { MAA, MAABV }\end{array}$ & 12 \\
\hline Thalassiosira angulata & $\begin{array}{l}\text { SL-2 } \\
\text { SL-1 }\end{array}$ & $\begin{array}{l}100,80,60,40,20 \\
20\end{array}$ & MA, SW, MAE, SWE & 11 \\
\hline Thalassiosira allenii & $\begin{array}{l}\text { SL-3 } \\
\text { SL-2 }\end{array}$ & $\begin{array}{l}100,80,60,40,20,100 \mathrm{~A}, 60 \mathrm{~A} \\
100\end{array}$ & MA, SW, MAE, SWE & 10 \\
\hline Chaetoceros gracile & SL-4 & $\begin{array}{l}100,80,60,40,20 \\
100 \mathrm{~A}, 60 \mathrm{~A}, 100 \mathrm{ABV}, 60 \mathrm{ABV}\end{array}$ & MA, SW, MAE, SWE & 11 \\
\hline Amphidinium carterae & SL-4 & $100,60,40,20$ & MA, SW, MAE, SWE & 13 \\
\hline Rhodomonas lens & SL-4 & $\begin{array}{l}100,80,60,40,20 \\
100 \mathrm{~A}, 60 \mathrm{~A}, 100 \mathrm{ABV}, 60 \mathrm{ABV}\end{array}$ & $\mathrm{MA}, \mathrm{SW}, \mathrm{MAE}$ & 10 \\
\hline Tetraselmis tetrathela & SL-4 & $100,80,60,40,20$ & MA, SW, MAE, SWE & 12 \\
\hline Chlorella sp. & SL-4 & $100,60,40,20$ & MA, SW, MAE, SWE & 13 \\
\hline Dunaliella tertiolecta & SL-4 & $\begin{array}{l}100,80,60,40,20 \\
100 \mathrm{~A}, 60 \mathrm{~A}, 100 \mathrm{ABV}, 60 \mathrm{ABV}\end{array}$ & MA, SW, MAE, SWE & 10 \\
\hline Isochrysis galbana & $\begin{array}{l}\text { SL-2 } \\
\text { SL-1 }\end{array}$ & $\begin{array}{l}100,80,60,40,20,100 \mathrm{~A}, 60 \mathrm{~A} \\
100\end{array}$ & MA, SW, MAE, SWE & 14 \\
\hline Emiliania huxleyi-1 & SL-4 & $\begin{array}{l}100,80,60,40,20 \\
100 \mathrm{~A}, 60 \mathrm{~A}, 100 \mathrm{ABV}, 60 \mathrm{ABV}\end{array}$ & MA, SW, MAE, SWE & 14 \\
\hline Emiliania huxleyi-2 & SL-4 & $\begin{array}{l}100,80,60,40,20 \\
100 \mathrm{~A}, 60 \mathrm{~A}, 100 \mathrm{ABV}, 60 \mathrm{ABV}\end{array}$ & MA, SW, MAE, SWE & 19 \\
\hline Synechococcus sp. & SL-4 & $100,80,60,40,20$ & MA, SW, MAE, SWE & 12 \\
\hline
\end{tabular}

* A and $\mathrm{ABV}$ suffixed to slag concentrations denote additional enrichments with nitrogen alone (A) and with a mixture of nitrogen, phosphorus and vitamins (ABV). 
phase and was inoculated into $100 \mathrm{~m} l$ of each medium. Two $40 \mathrm{~m} l$ aliquots of an inoculated medium were poured into two $50 \mathrm{~m} l$ culture tubes. The residual $20 \mathrm{~m} l$ aliquots were used to measure the initial phytoplankton chlorophyll $a$ concentration by both the acetone extraction method and the in vivo fluorescence method. The two culture tubes were sealed and mounted on a Model TRC slowly revolving rotor (Hario Inc.) and then incubated for 10 to 19 days under the conditions of $20^{\circ} \mathrm{C}$ and a 14:10 LD photocycled light of about $47 \mu \mathrm{E} \mathrm{m}^{-2}$ $\mathrm{s}^{-1}$. Therefore, the culture experiments were done either in duplicate and occasionally in triplicate.

After a gentle but thorough mixing at one or two day intervals about $3 \mathrm{~m} l$ subsamples were removed from each tube to a $10 \mathrm{~mm}$ glass cuvette to measure the chlorophyll $a$ concentration. The concentration was measured first on in vivo fluorescence (IVF) and then on an extract in $90 \%$ acetone solvent after filtration the same sample through a Whatman GF/F filter. IVF was measured with Hitachi Models F-1000 and F-2000 spectrofluorophotometers. The recorded IVF values for 1 to $2 \mathrm{~min}$ were averaged to obtain a mean value. The fluorescence of the extracted chlorophyll a was measured with a Hitachi 139 fluorometer following the technique described by Yentsch and Menzel. ${ }^{10)}$

The daily growth rate $\left(\mu \mathrm{day}^{-1}\right)$ of the phytoplankters was calculated from the increasing rate of chlorophyll $a$ by assuming an exponential growth rate:

$$
\mu=\left(\ln C_{1}-\ln C_{0}\right) /\left(t_{1}-t_{0}\right),
$$

where $C_{0}$ and $C_{1}$ are chlorophyll $a$ concentrations on $t_{0}$ day and $t_{1}$ day, respectively. Then $\mu$ could be converted into doublings per day $\left(\mu_{2}\right.$ day $\left.^{-1}\right)$ by using the formula:

$$
\mu_{2}=\mu / \ln 2 \text {. }
$$

\section{Results and Discussion}

\subsection{Chemical Characteristics of Slag}

The chemical composition of the slag used for the present experiments is given in Table 2. This slag con- tains some elements essential for algal growth such as $\mathrm{P}, \mathrm{Si}, \mathrm{Fe}$ and $\mathrm{Mn}$. Table 3 shows the data of chemical analysis of the slag solution prepared for growth experiments as well as the chemical analysis of natural filtered seawater and artificial seawater. It appears that there was not a notable difference in the chemical composition between the natural seawater and the artificial seawater, but there were differences between these and the slag solutions. For the first 10 days the slag solution SL-1 was made with a new slag. Then for the next 10 days solution SL-2 was made with the residue from the SL-1. Next, solution SL-3 was made with the residue from the SL-2. In addition to these three, SL-4 was prepared by mixing three similar solutions prepared with a new slag. As seen in Table 3 the concentrations of phosphorus in the slag solutions were $90-110 \mu \mathrm{g} / \mathrm{kg}$. These values were 11.3-13.8 times higher than in artificial seawater. The concentrations of most elements in the slag solutions were several times higher: Fe 1.1-3.9 times, Mn 62-70 times, Si 42.5-81.3 times, Ca 3.3-10.7 times and $\mathrm{Ba}$ 13.3-14.3 times. However, $\mathrm{Mg}$ in the slag solutions was $0.15-0.5$ times lower than in the artificial seawater. Compared to the Matsudaira medium, the concentrations of $\mathrm{P}$ and $\mathrm{Fe}$ in the slag solution were about 0.1 fold lower, those of $\mathrm{Co}$ and $\mathrm{Cu}$ were comparable, and those of $\mathrm{Mn}$ and $\mathrm{Si}$ were 2-10 times higher. This analytical technique using ICP defined the chemical elements, but not their compounds.

Table 2. Composition of chemical elements of the steelmaking slag. (\%)

\begin{tabular}{lc|ll}
\hline Element & $\%$ & Element & $\%$ \\
\hline $\mathrm{T}-\mathrm{Fe}$ & 20.17 & $\mathrm{SiO}_{2}$ & 8.56 \\
$\mathrm{FeO}$ & 16.35 & $\mathrm{P}_{2} \mathrm{O}_{5}$ & 2.27 \\
$\mathrm{Fe} \mathrm{O}_{3}$ & 7.63 & $\mathrm{MnO}$ & 3.50 \\
$\mathrm{M}-\mathrm{Fe}$ & 2.12 & $\mathrm{~T}-\mathrm{S}$ & 0.021 \\
$\mathrm{~T}-\mathrm{CaO}$ & 50.07 & $\mathrm{MgO}$ & 7.25 \\
$\mathrm{f}-\mathrm{CaO}$ & 18.21 & $\mathrm{Al}_{2} \mathrm{O}_{3}$ & 0.41 \\
$\mathrm{CaF}_{2}$ & 1.07 & $\mathrm{Na}_{2} \mathrm{O}$ & 5.80 \\
\hline
\end{tabular}

(T: total; f, free; M: metallic)

Table 3. Concentrations of major elements in steel-making slag solution (SL-1-SL-4), artificial seawater (MA) and natural seawater

\begin{tabular}{|c|c|c|c|c|c|c|}
\hline \multirow{2}{*}{ Element $(\mu \mathrm{g} / \mathrm{kg})$} & \multicolumn{4}{|c|}{ Slag solutions } & \multirow{2}{*}{ MA } & \multirow{2}{*}{ SW } \\
\hline & SL-1 & SL-2 & SL-3 & SL-4 & & \\
\hline $\mathrm{P}$ & 110 & 90 & 102 & 103 & 8 & 5 \\
\hline $\mathrm{Fe}$ & $\leqq 10$ & $\leqq 14$ & $\leqq 30$ & $<7$ & 3.6 & 3.1 \\
\hline $\mathrm{Mn}$ & 31 & 35 & 32 & 32 & 0.5 & 0.3 \\
\hline $\mathrm{Si}\left(\times 10^{3}\right)$ & 3.31 & 4.88 & 3.57 & 2.55 & 0.06 & 0.19 \\
\hline $\mathrm{Mg}\left(\times 10^{5}\right)$ & 6.11 & 4.42 & 1.85 & 3.00 & 12.3 & 12.5 \\
\hline $\mathrm{Ca}\left(\times 10^{5}\right)$ & 44.1 & 38.5 & 38.6 & 13.6 & 4.11 & 3.88 \\
\hline $\mathrm{Ba}$ & 215 & 200 & 204 & 210 & 15 & 21 \\
\hline $\mathrm{Al}$ & 4.2 & 10 & 12 & 2.3 & 1.6 & 2.9 \\
\hline $\mathrm{Co}$ & 0.7 & 1.6 & $<1$ & $<0.8$ & $\leqq 0.7$ & $\leqq 0.5$ \\
\hline $\mathrm{Zn}$ & 5.4 & $\sim 5$ & $\sim 5$ & 4.7 & 4.2 & -3.9 \\
\hline $\mathrm{Ni}$ & 2.2 & $\sim 2$ & $\sim 2$ & 2.1 & 1.8 & $\sim 1$ \\
\hline $\mathrm{Cu}$ & 2.1 & $\sim 2$ & $\sim 2$ & 1.6 & 1.2 & 7.4 \\
\hline $\mathrm{Cr}$ & $<0.5$ & $<0.5$ & $<0.5$ & $<0.5$ & $<0.5$ & $<0.5$ \\
\hline $\mathrm{Cd}$ & ND & ND & ND & ND & ND & ND \\
\hline
\end{tabular}
(SW) used in the enrichment culture experiments.

ND: not detected. 


\subsection{Relationship between Extracted Chlorophyll $a$ and In Vivo Fluorescence}

Certain biochemical constituents of phytoplankton can be used as an estimate of biomass. Chlorophyll $a$ is extracted with acetone is one of the most commonly used constituents. However, this pigment can be determined by only after tedious pretreatments. On the other hand, in vivo fluorescence (IVF) measurements should permit rapid determination of chlorophyll $a$ concentrations without filtering and extraction. ${ }^{1{ }^{1,12)}}$ However, the fluorescence yield (IVF per unit $\mathrm{Chl} a$ ) is known to vary with different species and different environments. ${ }^{13-24)}$ To determine the absolute amount of chlorophyll $a$ by IVF measurements, regression of the chlorophyll $a(\mathrm{Chl}$ a) values determined on the acetone extracts to IVF readings should be established. In this work, it was shown that, while individual organisms have different regression lines, the regressions always bear high and direct correlation coefficients (Table 4). In three plankters Synechococcus sp., D. tertiolecta and E. huxleyi, although the coefficients were significantly high, the linearity decreased with increasing chlorophyll $a$ concentration, which might be caused by increasing accessory pigments. The regression analyses in E. huxleyi were carried out for two different series started from inocula which were taken in different growth phases and yielded different regression lines carrying different correlation coefficients $(p<0.05)$. However, since no significant difference was observed in the intercepts, the amount of non-fluorescent fraction was likely to be relatively constant in cultures originating in the inocula of different ages. Based on these results, it can be reasonably assumed that the growth of each plankter can be monitored with IVF in any of the following culture experiments with an added steel-making slag solution.

Table 4. Regression analysis of in vivo fluorescence $(y)$ against the amount of chlorophyll $a$ in $90 \%$ acetone extraction $(x)$ for 12 phytoplankters.

\begin{tabular}{|c|c|c|c|}
\hline Species & Regression & $n$ & $r^{2}$ \\
\hline \multicolumn{4}{|l|}{ Bacillaliophyte (Diatoms) } \\
\hline Thalassiosira allenii & $y=3.24 x+2.5$ & 324 & 0.878 \\
\hline Thalassiosira angulata & $y=1.42 x+115.4$ & 240 & 0.885 \\
\hline Chaetoceros gracile & $y=0.20 x+3.1$ & 299 & 0.885 \\
\hline Skeletonema costatum & $y=0.52 x+3.9$ & 316 & 0.759 \\
\hline \multicolumn{4}{|l|}{ Dinophyte } \\
\hline Amphidinium carterae & $y=0.44 x+1.9$ & 145 & 0.874 \\
\hline \multicolumn{4}{|l|}{ Cryptophyte } \\
\hline Rhodomonas lens & $y=4.44 x-26.1$ & 260 & 0.892 \\
\hline \multicolumn{4}{|l|}{ Chlorophyte } \\
\hline Tetraselmis tetrathela & $y=0.16 x+3.0$ & 144 & 0.830 \\
\hline Dunaliella tertiolecta & $y=2.17 x+259.3$ & 260 & 0.539 \\
\hline Chlorella sp. & $y=0.64 x+4.4$ & 145 & 0.760 \\
\hline \multicolumn{4}{|l|}{ Haptophyte } \\
\hline Isochrysis galbana & $y=7.40 x+963.4$ & 119 & 0.810 \\
\hline Emiliania huxleyi-1 & $y=1.34 x+2.1$ & 268 & 0.560 \\
\hline Emiliania huxleyi-2 & $y=2.37 x+0.6$ & 261 & 0.706 \\
\hline \multicolumn{4}{|l|}{ Cyanophyte } \\
\hline Synechococcus sp. & $y=0.03 x+2.6$ & 145 & 0.359 \\
\hline
\end{tabular}

$n=$ number of samples, $r^{2}=$ correlation coefficient.
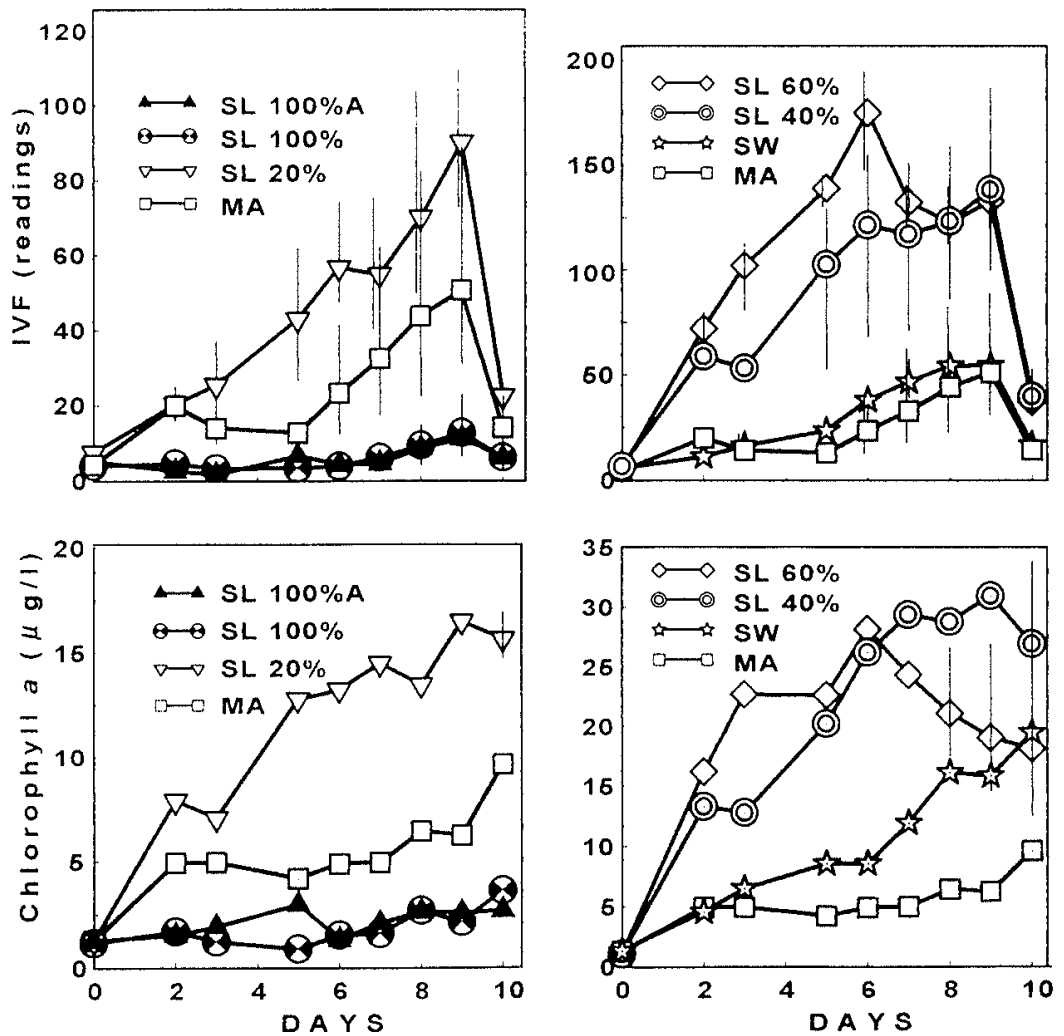

Fig. 1. Examples showing time courses of chlorophyll $a$ concentration (top) and readings of IVF (bottom) of Thalassiosira allenii in batch cultures enriched or not enriched with slag solution at different concentrations. MA and SW in control denote respectively artificial seawater and natural seawater. SL- $100 \%$ denotes additional enrichments with $\mathrm{NO}_{3}$ alone. 


\subsection{Daily Change of IVF under Enrichment with Slag Solution}

The IVF in the cultures enriched with the slag solution at different concentrations was measured every day or every other day. The growth of all the species examined was enhanced by enrichment. The typical time courses of those are shown in Fig. 1. The shape of the growth curves of any one species, for example, the inclination of the initial slope and time duration to reach the maximum density, was different under different enrichment conditions. The maximum levels of IVF attained did not remain constant, but usually fluctuated daily or decreased rapidly. Therefore, the growth rates (doublings day ${ }^{-1}$ ) were calculated in two ways. In the first, the growth rates were calculated for every interval over a 10 day incubation period. In the second, a single growth rate was calculated between the initial and the maximum values. The largest value obtained by the former calculations indicates the maximum instantaneous rate and usually occurred in the early stage of the incubation periods. The levels of the maximum yield did not significantly correlate with the maximum growth rates. However, the higher maximum yields were obtained after longer persistency of positive growth.

\subsection{Average Yields of IVF}

As mentioned above, it was quite difficult to express the growth ability of any one phytoplankter under different enrichment conditions by a single determination made on a fixed day. Therefore, to compare such a variable growth ability in a simple way, an average yield of IVF was determined by calculating the arithmetic mean of all the measurements made from the 2nd or 3rd day to the final day for each enrichment condition, which were made in duplicate or triplicate. The results are shown in Fig. 2. For $T$. angulata and $A$. carterae, the average IVF yields tended to be larger with increasing concentration of the slag solution (Fig. 2(a)). Except for an average IVF value observed in the $T$. angulata cultures enriched with 20 and $40 \%$ slag solution, the average yields of the enriched cultures were always significantly higher than those of the controls (F-test). This result suggests that these organisms prefer a high concentration of slag solution.

S. costatum grew significantly better under enrichment with the slag solution. The highest yield occurred at $80 \%$ slag solution and growth at $100 \%$ slag solution was observed to be slightly lower than this, while the latter was still higher than that in artificial seawater used as control. Similar results were also found in $T$. allenii, I. galbana and Chlorella sp. (Fig. 2(b)).

The concentration of the slag solution for optimal growth in C. gracile, $R$. lens and E. huxleyi was 20 $60 \%$, which was lower than in the other species described above (Fig. 2(c)). There were no significant differences in growth at higher concentrations of the slag solution $(80-100 \%)$ and in artificial seawater used as control. The experiments with $E$. huxleyi were repeated twice. Different results in the average yield were obtained, while the optimal growth was observed equally at lower concentrations $(20-40 \%)$. In some cases, though statistically not significant, the yields with enrichment at certain concentration were lower than in artificial seawater as control.

The growth of $D$. tertiolecta was enhanced by enrichment at any concentration. However, since the growth rate in artificial seawater used as control was also high, there was not significant difference in the average yield
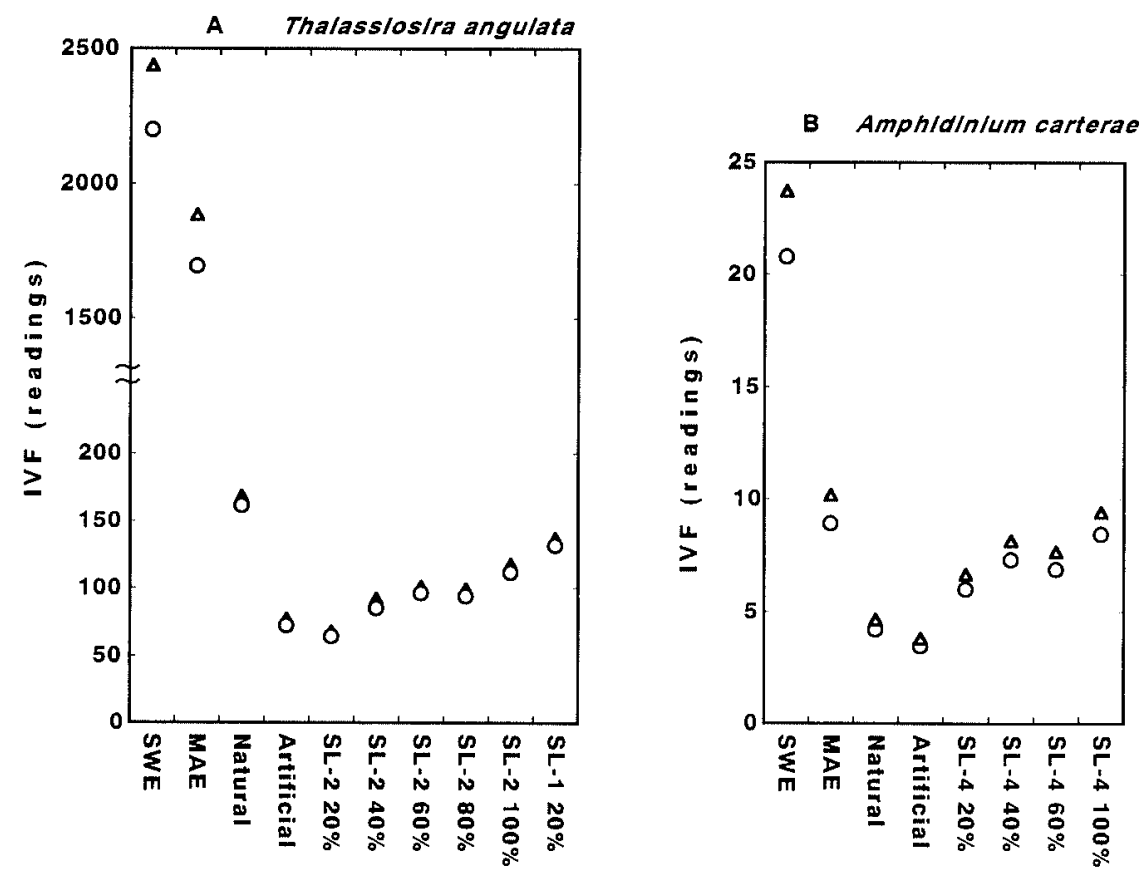

Fig. 2. Average yields in IVF of 12 phytoplankters in batch cultures enriched or not enriched with slag solution at different concentrations. The yields were expressed by arithmetic means of several measurements made from the 2 nd day $(O)$ or 3 rd day $(\triangle)$ to the final day.

2(a). Organisms which grow best under the highest concentration of slag solution (Group I). A: Thalassiosira angulata; B: Amphidinium carterae. 

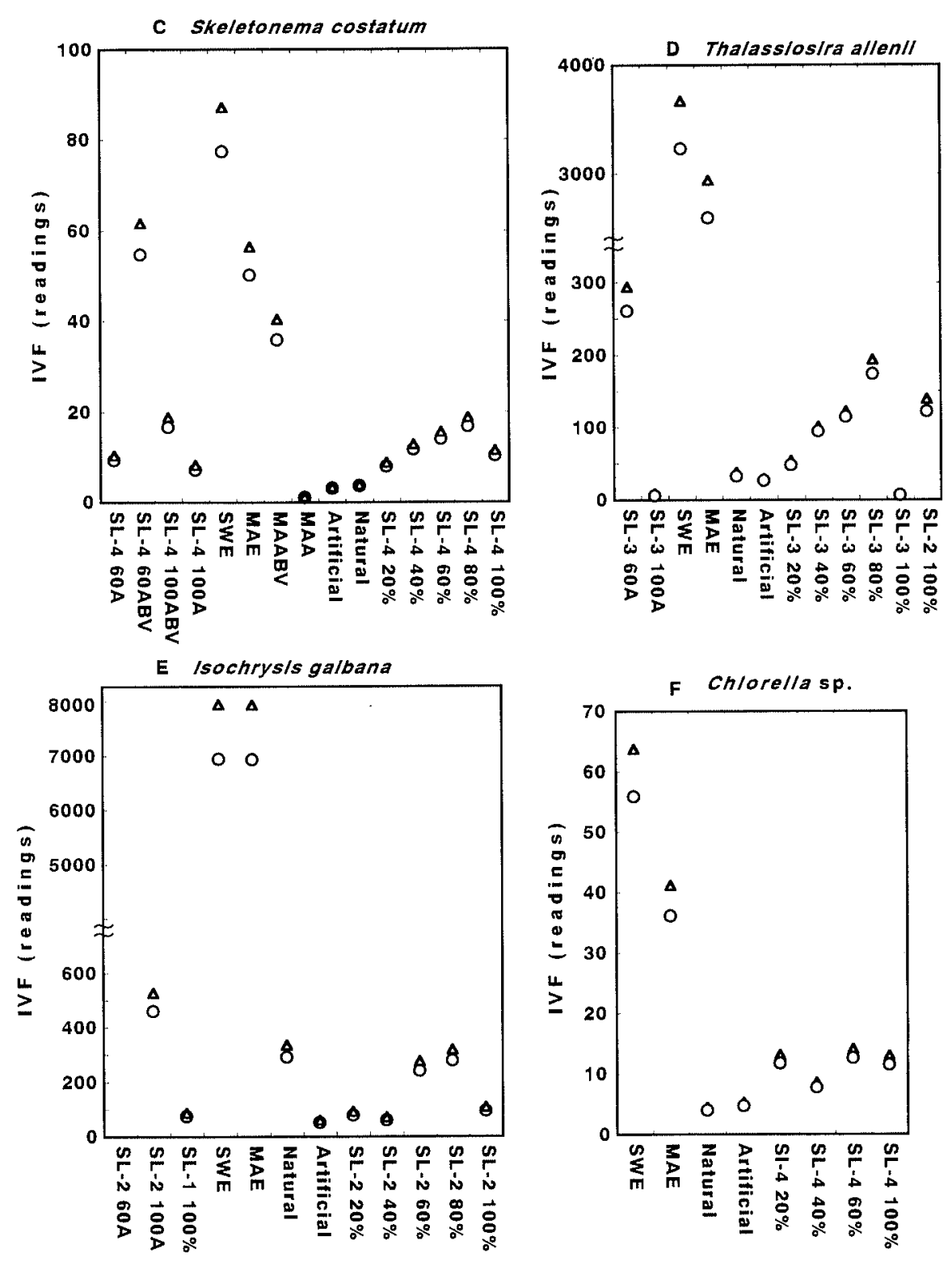

Fig. 2(b). (continued). Organisms which grow best in an intermediate concentration of slag solution, but not in $100 \%$ slag solution (Group II). C: Skeletonema costatum; D: Thalassiosira allenii; E: Isochrysis galbana; F: Chlorella sp.

between the enriched cultures and the control (Fig. 2(d)). This suggests that this alga is capable of moderate growth at a lower nutrient concentration. The growth of T. tetrathela in 80 and $100 \%$ slag solutions was weakly positive and that at lower concentration $(20-60 \%)$ was merely moderate, so that there was again no significant difference among different concentrations. The manners of response to the enrichment of Synechococcus sp. had a similar tendency to those of $D$. tertiolecta and $T$. tetrathela (Fig. 2(d)).

The growth enhancement of different lots of slag solution which contained elements at slightly different concentrations was compared for three phytoplankters. The results obtained were variable. Under $20 \%$ enrichment, T. angulata grew better in Lot SL-1 than in Lot SL-2. T. allenii grew better in $100 \%$ of Lot SL-2 than in $100 \%$ of Lot SL-3. However, the growth of $I$. galbana under $100 \%$ enrichment was little different between Lots SL-2 and SL-3.

\subsection{Effect of Additional Nutrients}

When a nitrogen source $\left(\mathrm{NO}_{3}^{-}\right)$was added to the cultures enriched with slag solution, the average IVF yields were almost always higher than without $\mathrm{NO}_{3}^{-}$. However, the results were again variable among lots and phytoplankters. Thus $T$. allenii, $R$. lens, I. galbana and $D$. tertiolecta tested in a $60 \%$ solution, and C. gracile, $S$. costatum, R. lens, I. galbana and D. tertiolecta in a $100 \%$ solution grew better when $\mathrm{NO}_{3}^{-}$was added. The only exception was $E$. huxleyi, which grew slower with $\mathrm{NO}_{3}^{-}$than in a slag solution without nitrogen. By further enrichment with a mixture of nitrogen, phosphorus $\left(\mathrm{PO}_{4}^{3-}\right)$ and vitamins (Vitamin Mixture 8 of Provasoli's formula) the growth of $D$. tertiolecta and $R$. lens in both 60 and $100 \%$ solutions and the growth of $S$. costatum and C. gracile in $60 \%$ solution were further enhanced. However, the growth rate of the latter two species was not enhanced by the addition of the mixture 

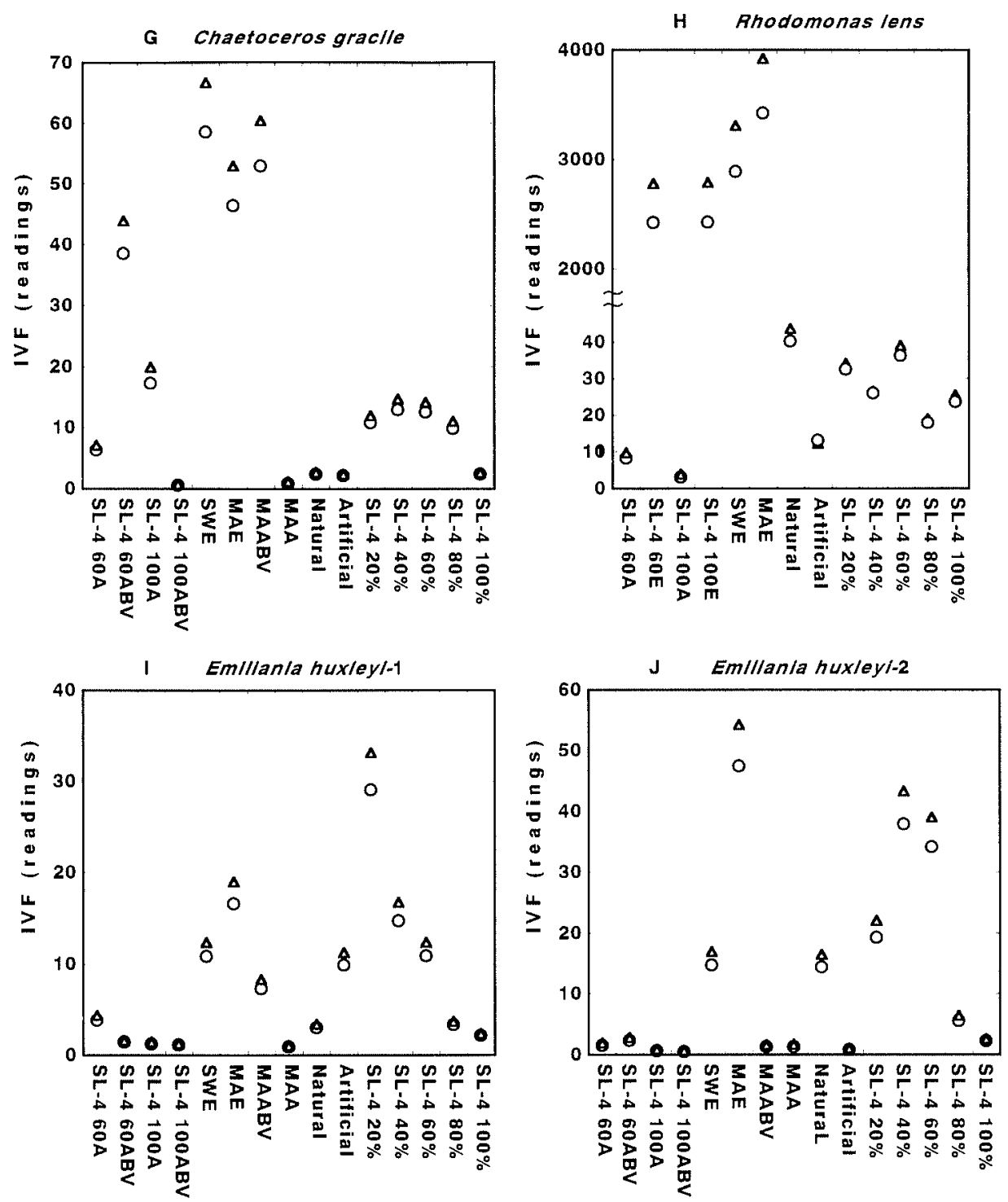

Fig. 2(c). (continued). Organisms which grow best under lower concentration of slag solution and are inhibited under concentrations higher than $60 \%$ (Group III). G: Chaetoceros gracile; H: Rhodomonas lens; I, J: Emiliania huxleyi.

with $100 \%$ solution. The exceptional species was again E. huxleyi, which grew slower under further addition of the mixture.

These results indicate that, except for a few species, the growth enhancement of slag solution can almost always be strengthened by further addition of a nitrogen source alone or by a mixture of nitrogen, phosphorus and vitamins. Nevertheless, the growth enhancement promoted by these enrichments never exceeded that achieved in an ideal culture medium.

\subsection{Types of Growth Enhancement}

According to the manner of response to the enrichment with slag solution at different concentrations, the organisms can be classified into the following four groups: (I) the organisms which grow best under the highest enrichment concentration $(100 \%)$, (II) those which grow best in an intermediate enrichment concentration, but not in $100 \%$ enrichment concentration, (III) those which grow best under a lower enrichment concentration, and which are inhibited under enrichment concentrations higher than $60 \%$, (IV) those which grow better under enrichment, regardless of the concentration (Table 5). Three among the four diatom species belong to Groups I and II. Dinoflagellate $A$. carterae also belongs to Group I.

The level of the maximum yields, which was determined by the enrichment concentrations, almost always showed a similar tendency with the levels of the average yields. However, as mentioned above, culture ages when the maximum yields appeared were clearly different among the enrichment concentrations. The level of the instantaneous growth rates between media enriched with slag solution at different concentrations or in another media prepared by the Matsudaira's formula also did not appear to make much difference.

\section{Concluding Remarks}

Our experiments show that diatoms grew significantly better under enrichment with the slag solution rather than another phytoplankton. Muggli and Harrison ${ }^{25}$ 

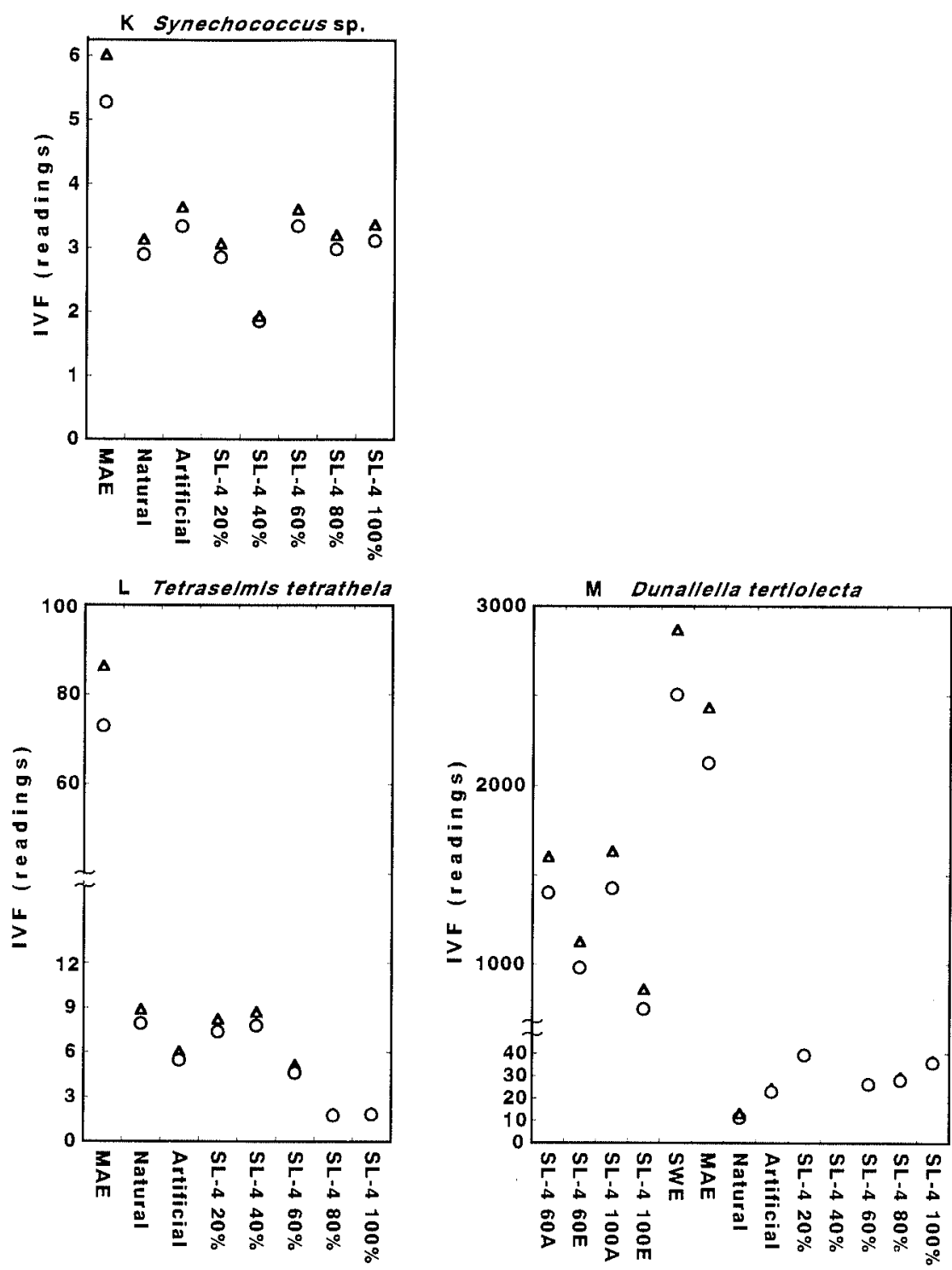

Fig. 2(d). (continued). Organisms which grow better under enrichment with slag solution, regardless of the concentration (Group IV). K: Synechococcus sp.; L: Tetraselmis tetrathela; M: Dunaliella tertiolecta.

Table 5. Four groups of phytoplankters classified by growth responses to enrichment with slag solution at different concentrations.

Group (I): Organisms which grow best under the highest concentration of slag solution.

Thalassiositra angulata (Bacillaliophyte)

Amphidinium carterae (Dinophyte)

Group (II): Organisms which grow best in an intermediate concentration of slag solution, but not in $100 \%$ slag solution. Skeletonema costatum (Bacillaliophyte)

Thalassiosira allenii (Bacillaliophyte)

Chlorella sp. (Chlorophyte) Isochrysis galbana (Haptophyte)

Group (III): Organisms which grow best under a lower concentration of slag solution, and which are inhibited under concentration higher than $60 \%$.

Chaetoceros gracile (Diatom)

Rhodomonas lens (Cryptophyte)

Emiliania huxleyi (Haptophyte)

Group (IV): Oraganisms which grow better under enrichment with slag solution, regardless of the concentration.

Dunaliella tertiolecta (Chlorophyte)

Tetraselmis tetrathela (Chlorophyte)

Synechococcus sp. (Cyanophyte) have pointed out that diatoms grew significantly faster than coccolithphores with $\mathrm{Fe}$ addition. It can therefore presume that $\mathrm{Fe}$ in slag extract may be important in growing marine phytoplankton. Diatoms were more strongly enhanced by iron than were small organisms, and large cells were more negatively affected by low levels of iron than were small cells. ${ }^{3,26)}$ Bruland et al. have pointed out that synergistic and antagonistic interactions between multiple trace metals can be very important in nature. ${ }^{27)}$ Cases have been presented showing the possible importance of a combination of bioactive trace metals. ${ }^{27-29)}$ The slag solution used in our experiments contained multiple trace metals. Although it was not clear which elements had strongly effected the phytoplankton growth, the slag solution apparently enhanced the growth of the phytoplankton tested. These results suggest that the slag solution could be utilized as nutrients in intensive culture of algae. The response of plankters for the slag enrichment were observed to be different. The slag enrichment to the mixed phytoplankton populations in nature would have an effect on alternation in species composition as 
well as community production. However, it would hardly cause the environmental change in species composition by enrichment with slag solution.

The growth enhancement by addition of the slag solution was strengthened for almost all phytoplankters except $E$. huxleyi by the simultaneous addition of either only nitrogen source or a mixture of nitrogen, phosphorus and vitamins. Nevertheless, the resultant growth was always lower than those in a culture medium enriched with an ideal nutrient mixture according to the Matsudaira's formula. In ecological considerations this indicates that enrichment with slag solution along with nitrogen would hardly cause the red tide in nature.

\section{Acknowledgments}

We are grateful to Mrs. Wang Xue-Lan for her technical assistance given during the experiments and Ms. Anne Thomas for her correction of our English. This work was partially funded by the Mitsubishi Foundation.

\section{REFERENCES}

1) K. Banse: Limnol. Oceanogr., 36 (1991), 1886.

2) H. J. W. De Baar, A. G. J. Buma, R. F. Nolting, G. C. Cadee, G. Jacques and P. J. Treguer: Mar. Ecol. Prog. Ser., 65 (1990), 105.

3) J. H. Martin and S. E. Fitzwater: Nature, 331 (1988), 341.

4) J. H. Martin, R. M. Gordon, S. E. Fitzwater and W. W. Broenkow: Deep-Sea Res., 36 (1989), 649.

5) J. H. Martin, R. M. Gordon and S. E. Fitzwater: Nature, 345 (1990), 156

6) J. H. Martin, R. M. Gordon and S. E. Fitzwater: Limnol. Oceanogr., 36 (1991), 1793.
7) J. H. Martin: Primary Productivity and Biogeochemical Cycles in the Sea, ed. by P. G. Falkowski and A. D. Woodhead, Plenum Press, New York, (1992), 123

8) M. Tokuda: The 135th Nishiyama Kinen Gijutsu Kouza, ed. by ISIJ, Tokyo, (1990), (in Japanese), 143.

9) Y. Nakamura: Bull. Jap. Soc. Microb. Ecol., 6 (1991), 71.

10) C. S. Yentsch and D. W. Menzel: Deep-Sea Res., 10 (1963), 221

11) C. J. Lorenzen: Deep-Sea Res., 13 (1966), 223.

12) J. D. H. Strickland: Deep-Sea Res., 15 (1968), 225.

13) A. E. Alpine and J. E. Cloern: J. Plankton Res., 7 (1985), 381.

14) D. Blasco: Inv. Pesq., 37 (1973), 533.

15) J. J. Cullen: Can. J. Fish. Aquat. Sci., 39 (1982), 791.

16) G. P. Harris: J. Plankton Res., 2 (1980), 109.

17) S. I. Heaney: Freshwater Biol., 8 (1978), 115.

18) D. A. Kiefer: Mar. Biol., 22 (1973a), 263.

19) D. A. Kiefer: Mar. Biol., 23 (1973b), 39.

20) M. E. Loftus and H. H. Seliger: Chesapeake Sci., 16 (1975), 79.

21) E. Sakshaug, S. Demers and C. M. Yentsch: Mar. Ecol. Prog. Ser., 41 (1987), 275.

22) R. E. Slovacek and P. J. Hannan: Limnol. Oceanogr., 22 (1977), 919.

23) A. Taniguchi, E. Hamada, M. Okazaki and Y. Naito: Mem. Nat. Inst. Polar Res. Spec. Issue, 44 (1986), 3.

24) T. Vandevelde and L. Legendre: Can. J. Fish. Aquat. Sci., 45 (1988), 1508.

25) D. L. Muggli and P. J. Harrison: J. Exp. Mar. Biol. Ecol., 212 (1997), 225

26) R. J. M. Hudson and F. M. M. Morel: Limnol. Oceanogr., 35 (1990), 1002

27) K. W. Bruland, J. R. Donat and D. A. Hutchins: Limnol. Oceanogr., 36 (1991), 1555.

28) S. A. Huntsman and W. G. Sunda: The Physiological Ecology of Phytoplankton, ed. by I. Morris, Blackwell Scientific Publications, London, (1980), 285.

29) M. M. Morel, R. J. M. Hudson and N. M. Price: Limnol. Oceanogr., 36 (1991), 1742. 